\title{
Analysis of dynamic meshing characteristic of planetary gear transmission in wind power increasing gearbox
}

\author{
Jungang Wang ${ }^{1, *}$, Ruina $\mathrm{Mo}^{2}$, Zhaoping Tang ${ }^{1}$ \\ ${ }^{1}$ School of Mechanotronics \& Vehicle Engineering, East China Jiaotong University, Nanchang 330013, China \\ ${ }^{2}$ School of Basic Science, East China Jiaotong University, Nanchang 330013, China
}

\begin{abstract}
Dynamic behavior of planetary gear's tooth contact surface in the different location can better conform operation condition comparing to the general gear pair. Nonlinear finite element algorithm was derived according to the basic control equation of contact dynamics. A finite element model of planetary gear transmission in wind power increasing gearbox was proposed considering different meshing locations based on nonlinear finite element solution. The characteristics of stress distribution at different meshing positions were analyzed. A simulation of the meshing process was conducted using finite element analysis. It was shown that node stresses of external meshing planetary gear varied significantly at different position. The analysis provides some useful insights into the performance of planetary gear's tooth contact surface.
\end{abstract}

Key words: Equivalent stress, planetary gear, finite element analysis, analysis of stress distribution

\section{Introduction}

Planetary gear transmission is used widely, which use more than one planet gear to support load on the structure of the average allocation. Compared with the general gear meshing transmission, planetary gear exists internal meshing and external meshing at the same time. There is a vibration and impact of planetary gears in the process of the actual work. It is very difficult to test the actual work condition of structure strength. With the help of simulation analysis, the danger zone load characteristics of planetary gears can be obtained. However, few papers are concerned with the internal meshing and external meshing planetary gear pair meshing process and the research is not enough in-depth. The research on the contact problems of gear drives was reported by many investigators. Lin et al. [1] proposed a method for mesh generation to analyze meshing stiffness under static loading. A three-dimensional $\mathrm{r}$ model of helical gears was presented by Eritenel [2]. Cai et al. [3] investigated the dynamic behavior of a gear-bearing system considering the nonlinear gear meshing force effect. Chen et al. [4] carried out a study on the contact stress of gear pairs with non-parallel axes by means of finite element method. Zhang et al. [5] carried out a study on the bending fatigue failure of a bevel gear. Palermo et al. [6] proposed a contact element to simulate global dynamic behavior of gear assemblies. The tooth engagement behavior of beveloid gears was analyzed through formulating a pitch cone design theory by Zhu
[7]. Tamarozzi et al. [8] analyzed flexible multibody model of two meshing gears by numerical simulation. Dong et al. [9] proposed a new method to study the contact fatigue life of gear-bearing system of a wind turbine. Li et al. [10] studied the influence of addendum on bending strength and tooth contact strength of spur gears. Khabou et al. [11] investigated the dynamic performance of a spur gear reducer in transient regime.

Few reports about stress distribution of external meshing gear tooth profiles in different mesh position using finite element model are concerned. Lumped parameter model and the finite element analysis (FEA) just for teeth were adopted in references [1-10] to analyze the dynamic characteristics of meshing contact transmission with parallel axis. However, the FEA just for teeth is not fully conformed to actual condition of planetary gear transmission in engineering application, leading to that the actual condition cannot be reflected in full scale. A finite element model with different meshing locations of planetary gear pairs is limitedly reported.

Differences of stress distribution on tooth profile at different meshing positions of planetary gear transmission in wind power increasing gearbox were investigated in this paper. The finite element model of entire gear with both external and internal meshing was established. The entire gears were built in order to simulate dynamic process of planetary gear pair more accurately. Boundary and initial conditions of the finite element model were decided. Load was applied and working parameters were set. The characteristics of

\footnotetext{
* Corresponding author: $\underline{\text { sduhys@ } 163 . c o m}$
} 
stress distribution of external meshing planetary gears were obtained. The stress distribution rule in the different areas of the gear tooth profile of the external gear planetary was studied in detail.

\section{Nonlinear finite element solution to the contact problem}

According to the basic control equation of contact dynamics, the nonlinear differential equation of the contact system can be derived from the Hamilton variation principle:

$$
M \ddot{X}+D \dot{X}+r(X)=f(t)
$$

Where $D, M, r(X)$ and are damping matrix, mass matrix and nonlinear matrix of the contact system, $\ddot{X}, \dot{X}$ and $X$ are acceleration vector, velocity vector and displacement vector of the system, $f(t)$ is load vector.

Under the known initial conditions, convert equation (1) into the nonlinear algebraic equation set in the incremental form, and then use Newton-Raphson for iterative solution. Assuming that at the time of the $t_{n}$, all variable values are known, and determines the displacement $X^{*}=X_{t_{n}+\Delta t_{n}}$ at the time of $t_{n}+\Delta t_{n}$.

The unbalanced force of the system can be obtained from equation (1):

$$
\widetilde{f}\left(X^{*}\right)=M \ddot{X}+D \dot{X}+r(X)-f(t)
$$

According to equation (2), the values at the time of $t_{n}+\Delta t_{n}$ and in the (i-1) step of iterative process can be represented by:

$$
\widetilde{f}\left(U_{t_{n}+\Delta t_{n}}^{(i-1)}\right)=M \ddot{U}_{t_{n}+\Delta t_{n}}^{(i-1)}+D \dot{U}_{t_{n}+\Delta t_{n}}^{(i-1)}+r U_{t_{n}+\Delta t_{n}}^{(i-1)}-f_{t_{n}+\Delta t_{n}}
$$

The expansion of equation (4) can be obtained from Taylor series:

$$
\tilde{f}\left(X^{*}\right)=\tilde{f}\left(X_{t_{n}+\Delta_{n}}^{(i-1)}\right)+\left(\left.\frac{\partial \tilde{f}}{\partial X}\right|_{X_{t_{n}+\Delta_{n}}^{(i-1)}}\right)\left(X^{*}-X_{t_{n}+\Delta_{n}}^{(i-1)}\right)+O\left(\left\|X^{*}-X_{t_{n}+\Delta_{n}}^{(i-1)}\right\|^{2}\right)
$$

The displacement increment is represented by:

$$
\Delta X^{(i-1)}=X_{t_{n}+\Delta t_{n}}^{(i)}-X_{t_{n}+\Delta t_{n}}^{(i-1)}
$$

From (5) and the second order small quantities in the ignored equation (4), the following expression can be obtained:

$$
\Delta X^{(i-1)}=-\left(\left.\frac{\partial \tilde{f}}{\partial X}\right|_{X_{t_{n}+\Delta t_{n}}^{(i-1)}}\right)^{-1} \widetilde{f}\left(X_{t_{n}+\Delta t_{n}}^{(i-1)}\right)
$$

From equations (5) and (6), the approximate value of the displacement $X$ at the time of $t_{n}+\Delta t_{n}$ can be obtained:

$$
X_{t_{n}+\Delta t_{n}}^{(i)}=X_{t_{n}+\Delta_{n}}^{(i-1)}-\left(\left.\frac{\tilde{\partial f}}{\partial X}\right|_{U_{t_{n}+\Delta_{n}}^{(i-1)}}\right)^{-1} \widetilde{f}\left(X_{t_{n}+\Delta_{n}}^{(i-1)}\right) \quad(i=1,2, \cdots)
$$

$\widetilde{f}\left(X_{t_{n}+\Delta t_{n}}^{(i-1)}\right)$ in the equation (7) can be directly calculated from equation (3).

Use Newmark to calculate Jacobian matrix $\partial \tilde{f} / \partial X$ in equation (7) :

$$
\left.\frac{\partial \tilde{f}(X)}{\partial U}\right|_{X_{t_{n}+\Delta_{n}}^{(i-1)}}=\left.M \frac{X}{\partial X}\right|_{X_{t_{n}+\Delta_{n}}^{(i-1)}}+\left.D \frac{X}{\partial X}\right|_{X_{t_{n}+\Delta_{n}}^{(i-1)}}+\left.\frac{\partial r(X)}{\partial X}\right|_{X_{t_{n}+\Delta_{n}}^{(i-1)}}
$$

In Newmark algorithm, the expressions of the increments of speed and acceleration:

$$
\begin{gathered}
\dot{X}_{i+1}=\dot{X}_{i}+(1-\gamma) \Delta t_{i} \ddot{X}_{i}+\gamma \Delta t_{i}\left(\frac{X_{i+1}-X_{i}-\dot{X}_{i} \Delta t_{i}}{\beta \Delta t_{i}^{2}}-\left(\frac{1}{2 \beta}-1\right) \ddot{X}_{i}\right) \\
\ddot{X}_{i+1}=\frac{X_{i+1}-X_{i}-\dot{X}_{i} \Delta t_{i}}{\beta \Delta t_{i}^{2}}-\left(\frac{1}{2 \beta}-1\right) \ddot{X}_{i}
\end{gathered}
$$

Where: $\gamma$ and $\beta$ are calculation coefficients.

Substitute equations (9) and (10) into equation (8) and simplify it, and then obtain:

$$
\left.\frac{\partial \tilde{f}(X)}{\partial X}\right|_{X_{t_{n}+\Delta_{n}}^{(i-1)}}=\frac{1}{\beta \Delta t_{n}^{2}} M+\frac{\gamma}{\beta \Delta t_{n}} D+\left.\frac{\partial r(X)}{\partial X}\right|_{X_{t_{n}+\Delta_{n}}^{(i-1)}}
$$

Substitutes equations (3) and (11) into equation (6) and obtains:

$$
\begin{gathered}
\left(\frac{1}{\beta \Delta t_{n}^{2}} M+\frac{\gamma}{\beta \Delta t_{n}} D+\left.\frac{\partial r(X)}{\partial X}\right|_{X_{t_{n}+\Delta_{n}}^{(i-1)}}\right) \Delta X^{(i-1)}= \\
f_{t_{n}+\Delta t_{n}}-M \ddot{X}_{t_{n}+\Delta t_{n}}^{(i-1)}-D \dot{X}_{t_{n}+\Delta t_{n}}^{(i-1)}-r X_{t_{n}+\Delta t_{n}}^{(i-1)} \\
\ddot{X}_{t_{n}+\Delta t_{n}}^{(i-1)}=\frac{X_{t_{n}+\Delta t_{n}}^{(i-1)}-X_{t_{n}}-\dot{X}_{t_{n}} \Delta t_{n}}{\beta \Delta t_{n}^{2}}-\left(\frac{1}{2 \beta}-1\right) \ddot{X}_{t_{n}} \\
\dot{X}_{t_{n}+\Delta t_{n}}^{(i-1)}=\dot{X}_{t_{n}}+(1-\gamma) \Delta t_{n} \ddot{X}_{t_{n}}+\gamma \Delta t_{n} \ddot{X}_{t_{n}+\Delta t_{n}}^{(i-1)}
\end{gathered}
$$

Where $\ddot{X}_{t_{n}}, \dot{X}_{t_{n}}$ and $X_{t_{n}}$ are the acceleration vector, the displacement vector, the speed vector and at the time of $t_{n}$. According to the displacement, velocity, acceleration and interpolation function of the calculated node point, physical quantities, such as internal displacement, strain, stress, can be obtained.

\section{Simulation of dynamic meshing contact}

The finite element model of the planetary gear pair was established, which had both the functions of inner and outer contact, as shown in Fig. 1. Boundary conditions parameters were specified. The finite element model with constraints setting was shown in Fig. 2. 


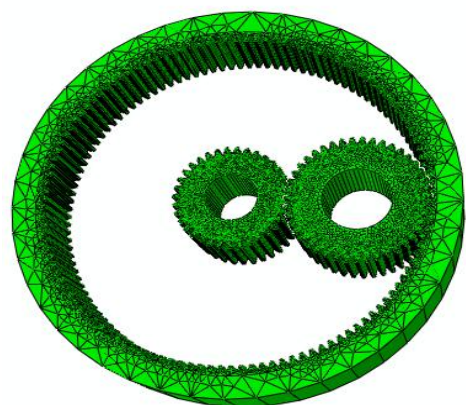

Fig. 1 A planetary gearing finite element model

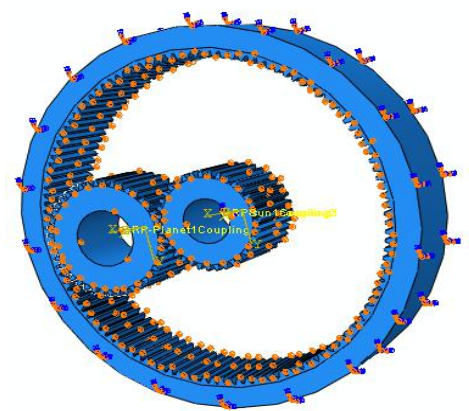

Fig. 2 Planetary gear pair with constraints

\subsection{Analysis of stress distribution around addendum}

The simulation of planetary gear pair was adopted to study dynamic behavior around addendum of external meshing planetary gear in meshing process. 5 nodes were chosen, numbered by 1, 2, 3, 4 and 5, respectively, as was shown in Fig.3 (a). Node 1 was on the meshing surface of addendum, and node 2 in the area near meshing surface. Node 3 was in the middle of addendum. Node 4 was in the area near non-meshing surface, while node 5 on the non-meshing surface of addendum. The time history curves of equivalent stresses of nodes shown in Fig.3 (a) were presented in Fig.3 (b), where it can be found that in meshing process the equivalent stress of each node increases first and then decreases, showing similar variation.

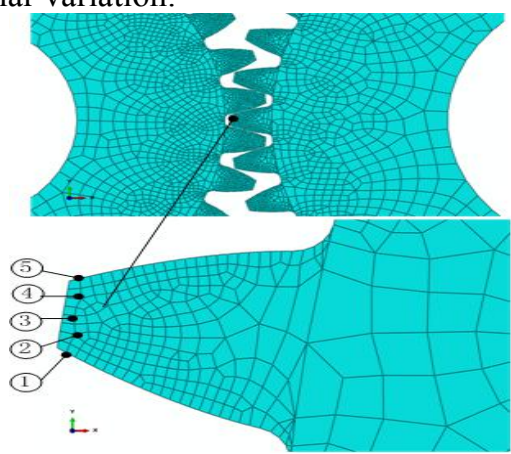

(a) Nodes around addendum of external meshing planetary gear

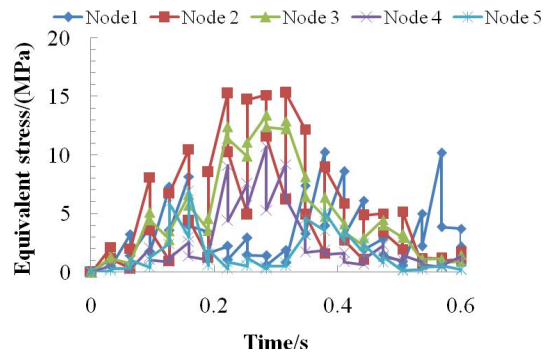

(b) Time history curves of equivalent stress of each node around addendum

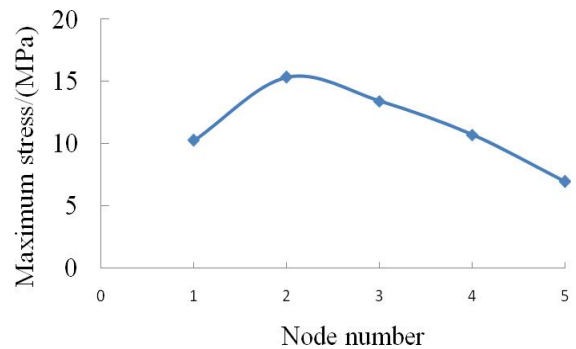

(c) Maximum equivalent stress of each node around addendum

Fig.3 Stress around addendum of external meshing planetary gear

The diagram was drawn taking each node shown in Figure 2(a) as abscissa, the maximum equivalent stress corresponding to each node as ordinate, as shown in Fig.3 (c). It could be known from both Fig.3 (c) and Fig.3 (a) that minimum stress was on the non-mating surface of the addendum. Less stress was in the nonengagement surface area close to the addendum. Greater stress was on the meshing surface of the addendum. Maximum stress was in the area close to the addendum mating surface. The maximum stress of node 1 was $33.06 \%$ smaller than that of node 2 , the maximum stress of node 2 was $14.26 \%$ greater than that of node 3 , the maximum stress of node 3 was $25.37 \%$ greater than that of node 4 , the maximum stress of point 4 was $54.51 \%$ greater than that of node 5 , and a difference between the minimum equivalent stress and the maximum was $54.82 \%$.

\subsection{Analysis of stress distribution around pitch circle}

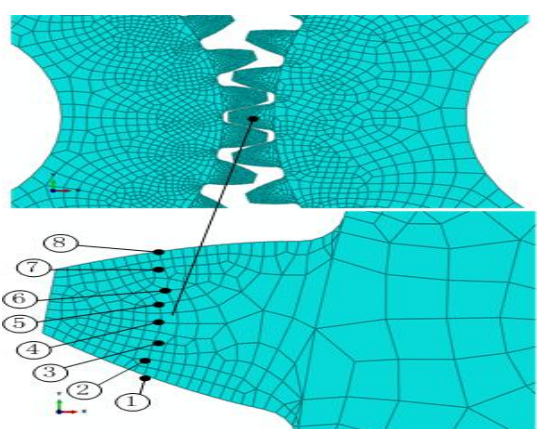

(a) Nodes of external meshing planetary gear's tooth profile around pitch circle 


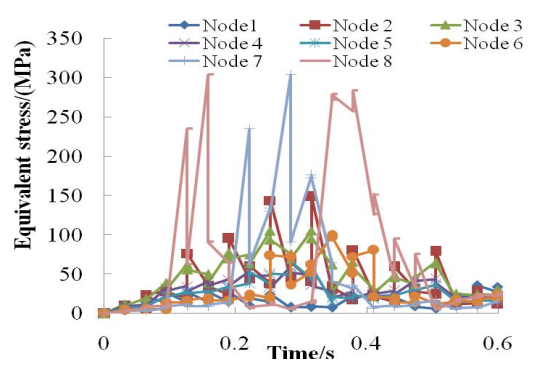

(b) Time history curves of equivalent stress

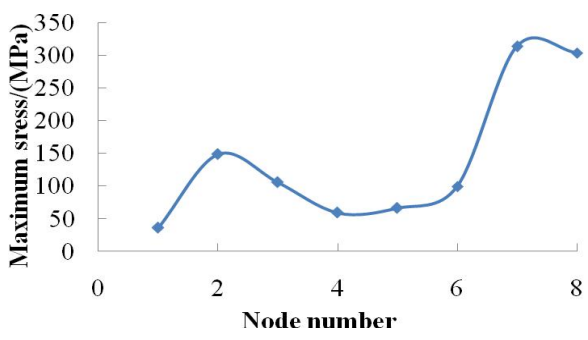

(c) Maximum equivalent stress of each node around pitch circle

Fig.4 Stress of external meshing planetary gear's tooth profile around pitch circle

The simulation of dynamic meshing process was adopted to study dynamic behavior around addendum of external meshing planetary gear. 8 nodes were chosen, numbered by 1, 2, 3, 4, 5, 6, 7 and 8, respectively, as was shown in Fig.4 (a). Node 1 was on the meshing surface of pitch circle, and node 2 in the area near meshing surface. Nodes 3 6 were in the middle of pitch circle. Node 7 was in the area near non-meshing surface, while node 8 was on the non-meshing surface of pitch circle. The time history curves of equivalent stresses of nodes shown in Fig.4 (a) were presented in Fig.4 (b), where it can be found that in meshing process the equivalent stress of each node increases first and then decreases, showing similar variation.

The maximum equivalent stress of each node around pitch circle was calculated, as was presented in Fig.4 (c), where abscissa was node number and ordinate was corresponding maximum equivalent stress. It can be shown in Fig.4 (c) combined with Fig.4 (a) that the maximum stress on the meshing surface of pitch circle is the smallest, while that in the area near non-meshing surface is the largest. The stresses in the middle of pitch circle are relatively small, and those on the non-meshing surface and in the area near meshing surface are larger. The maximum stress of node 1 is $75.82 \%$ less than that of node 2, which is $29.18 \%$ larger than that of node 3 , while maximum stress of node 7 is $68.39 \%$ larger than that of node 6 . The difference between minimum and maximum values of maximum equivalent stress is $88.53 \%$. The stresses of nodes at different position of addendum of external stress planetary gear vary significantly.

\subsection{Analysis of stress distribution around dedendum}

Considering the different features between pitch circle and dedendum, 6 nodes were chosen to study the dynamic behavior around dedendum, instead of 8 nodes. The nodes were numbered by $1,2,3,4,5$ and 6 , successively from meshing surface to non-meshing surface, as was shown in Fig.5 (a). Time history curves of equivalent stresses of nodes in Fig. 5 (a) were shown in Fig.5 (b) using finite element modeling. The equivalent stress around dedendum increases first, followed by decreasing.

The relationship between the number of node around dedendum (Fig.5 (a)) and corresponding maximum equivalent stress was obtained in Fig.5 (c). Significantly different from the law around pitch circle, the curve shows a "V" type. The maximum equivalent stress around dedendum decreases at first to $25.85 \mathrm{MPa}$ at the position of node 3 , from $56.51 \mathrm{MPa}$ at that of node 1 , and then increases continuously to $93.56 \mathrm{MPa}$ at the position of node 6 , which is also the largest maximum equivalent stress.

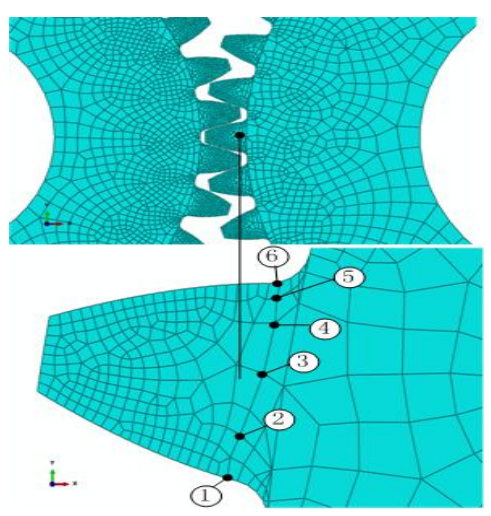

(a)Nodes of external meshing planetary gear's tooth profile around dedendum

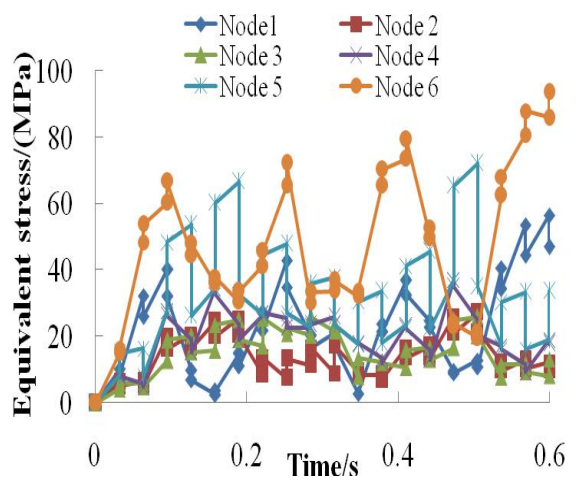

(b) Time history curves of equivalent stress

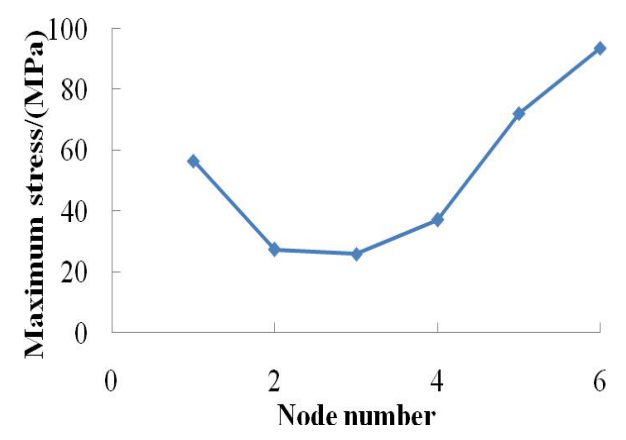

(c) Maximum equivalent stress 
Fig.5 Stress of external meshing planetary gear's tooth profile around dedendum

\section{Conclusion}

Stress at each node of external meshing planetary gear has the similar change rule on the time course, which increases first and then decreases in the dynamic meshing process. Characteristics of stress distribution differ from one another in different meshing areas of the external meshing planetary gear's tooth profile. The difference of the equivalent stress around addendum, pitch circle and dedendum is relatively significant from the mating surface to the non-mating surface. The maximum stress of nodes at different position of external stress planetary gear varies significantly. Around dedendum, the maximum equivalent stress is obtained on the non-mating surface, while the smallest value of it is in the middle of dedendum.

\section{Acknowledgement}

The authors would like to thank anonymous reviewers for their helpful comments and suggestions to improve the manuscript. This research was supported by the Natural Science Foundation of Jiangxi Province (Grant No. 20161BAB206153), and the National Natural Science Foundation of China (Grant No.51765020), and the National Natural Science Foundation of China(Grant No.51765015).

\section{References}

[1] Lin T, Ou H, Li R F. A finite element method for 3D static and dynamic contact/impact analysis of gear drives . Computer Methods in Applied Mechanics and Engineering, 2007, 196(9): 1716-1728.

[2] Eritenel T, Parker R G. An investigation of tooth mesh nonlinearity and partial contact loss in gear pairs using a lumped-parameter model. Mechanism and Machine Theory, 2012, 56:28-51.

[3] Cai W, Chang J. Bifurcation and chaos analysis of the porous squeeze film damper mounted gear-bearing system. Computers \& Mathematics with Applications, 2012, 64(5):798-812.

[4] Chen Y C, Liu C C. Contact stress analysis of concave conical involute gear pairs with non-parallel axes . Finite Elements in Analysis and Design, 2011, 47(4): 443-452.

[5] Chen Y C, Liu C C. Investigation on bending fatigue failure of a micro-gear through finite element analysis . Engineering Failure Analysis, 2013, 31: 225235.

[6] Palermo A., Mundo D., Hadjit R., Desmet W. Multibody element for spur and helical gear meshing based on detailed three-dimensional contact calculations . Mechanism and Machine Theory, 2013, 62:13-30.

[7] Zhu C C, Song C S, Lim T C, Peng T. Pitch cone design and influence of misalignments on tooth contact behaviors of crossed beveloid gears . Mechanism and Machine Theory, 2013, 59:48-64.

[8] Tamarozzi T, Ziegler P., Eberhard P, Desmet W. Static modes switching in gear contact simulation. Mechanism and Machine Theory, 2013, 63:89-106.

[9] Dong W B, Xing Y H, Moan T, Gao Z. Time domain-based gear contact fatigue analysis of a wind turbine drivetrain under dynamic conditions. International Journal of Fatigue, 2013, 48:133-146.

[10] Li S. Effect of addendum on contact strength, bending strength and basic performance parameters of a pair of spur gears . Mechanism and Machine Theory, 2008, 43:1557-1584.

[11] Khabou M T, Bouchaala N, Chaari F, Fakhfakh T, Haddar M. Study of a spur gear dynamic behavior in transient regime. Mechanical Systems and Signal Processing, 2011, 25(8):3089-3101. 